\title{
Diaries and diary techniques
}

\author{
David F Marks
}

Potential competing interests: The author(s) declared that no potential competing interests exist.

Diaries and diary techniques are a method for collecting information about temporal changes in experience and/or behaviour. 